\title{
Characterization of Experimental Rat Nephroblastoma and Its Cell Line
}

\author{
Yoji Nagashima, Yohei Miyagi, Kaoru Sumino, \\ Yoshinaru Ohaki, Makoto Umeda*, Mitsuo Oshimura $\dagger$ \\ and Kazuaki Misugi \\ Department of Pathology, Yokohama City University School \\ of Medicine, Yokohama 236, *Department of Cell Biology, \\ Kihara Institute for Biological Research, Yokohama City \\ University Yokohama 232, and †Laboratory of Molecular \\ and Cellular Genetics, Tottori University School of \\ Medicine, Yonago 683
}

Nagashima, Y., Miyagi, Y., Sumino, K., Ohaki, Y., Umeda, M., Oshimura, M. and Mrsugi, K. Characterization of Experimental Rat Nephroblastoma and Its Cell Line. Tohoku J. Exp. Med., 1992, 168 (2), 303-305 — Rat nephroblastoma (Wilms' tumor) was induced by transplacental administration of N-ethylnitrosourea (ENU). The induced renal tumors were histologically compatible with human nephroblastoma. A cultured cell line (ENU-T-1) established from a xenotransplant, showed similar morphological and biological features to cultured embryonal kidney cells. Introduction of normal human chromosome \#11 (\#11) bearing Wilms' tumor suppressor gene(s) (WT) suppressed colony-forming ability on soft agar plates (CFA) but tumorigenicity of ENU-T-1 was not affected. Whereas tumorigenicity of human nephroblastoma cell line, SK-NEP-1 was completely suppressed, CFA was unchanged. These facts indicated that pathogenenetic mechanism is different between human and experimental rat nephroblastomas. - nephroblastoma (Wilms' tumor) ; transplacental carcinogenesis ; cell line; chromosome transfer

Nephroblastoma (Wilms' tumor) is an embryonal tumor of the kidney, and hitologically similar to developing kidney. Cytogenetic and molecular biological studies have shown that deletion of WT gene(s) coded on \#11 is responsible to the pathogenesis (Wadey et al. 1990). Recently, one of the genes on \#11p13 band has been cloned (Call et al. 1990 ; Gessler et al. 1990), of which product is a transcription regulator with four zinc finger domains. On the other hand, Stanbridge and colleagues (Weissman et al. 1987 ; Dowdy et al. 1991) confirmed that introduction of \#11 or chromosome fragment including \#11p15 band caused suppression of tumorigenicity of a human nephroblastoma cell line.

In this presentation, we report establishment of an experimental system of rat nephroblastoma, and describe properties of the experimental tumor and estab-

Address for reprints : 3-9 Fukuura, Kanazawa-ku, Yokohama 236, Japan. 
lished cultured cell line.

\section{Materials and Methods}

Rat nephroblastomas were induced by intraperitoneal injection of N-ethylnitrosourea $(80 \mathrm{mg} / \mathrm{kg}$ rat) into pregnant Sprague-Dawley rats. The offsprings were sacrificed about 100 days after birth. The renal tumors were processed for morphological examination, transplantation into nude mouse and in vitro cultivation. The established cell line derived from the xenotransplant was named ENU-T-1, and was characterized morphologically and biochemically. For chromosome introduction to examine whether \#11 has suppressive effects on growth of the nephroblastoma cell line, (microcell $\times$ cell) fusion method was performed according to Weissman et al. (1987). As the recipient cell line, human nephroblastoma cell line, SK-NEP-1 was used as well as ENU-T-1. The hybrid cells conformed to have introduced extra-copy of \#11 were examined on growth characteristics and tumorigenicity in nude mice.

\section{RESULTS}

Renal tumors were observed in about $25 \%$ of the offspring rats. Histologically, these tumors were compatible with human classical nephroblastoma composed of blastomal and organoid elements. Ultrastructurally, these cells had poorly developed desmosomal junctions and surrounded with basement membrane. They contained abundant free ribosomes and a few mitochondria and endoplasmic reticulum, which indicates their functional immaturity.

Although the efforts to serially cultivate these tumors were not successful, a cultured cell line, ENU-T-1 was established from a xentransplanted tumor in a nude mouse, which showed marked histological atypism with lung metastasis. The cells showed epithelial pattern in vitro without dome formation. Histochemically, they showed activities of alkaline phosphatase and gammaglutamyltranspeptidase, which are characteristics of renal tubular epithelia. Immunofluorescent study revealed positivities both for cytokeratin and for vimentin. Ultrastructural examination showed luminal space between the cells associated with microvilli and desmosomal junctions. When transplanted into nude mice, ENU-T-1 formed tumors composed of immature epithelial cells with focal glandular arrangements.

The effects of introduction of \#11 into ENU-T-1 and human nephroblastoma cell line, SK-NEP-1 was observed. As the result of (microcell $\times$ cell) fusion, 4 and 3 microcell hybrids (ENU-T-\#11, SK-NEP-\#11) were obtained from ENU-T-1 and SK-NEP-1, respectively. The ENU-T-\#11 showed decreased colosy-forming ability (CFA), although tumorigenicity was sustained. Contrarily, SK-NEP-\#11 showed loss of tumorigenicity, whereas CFA was not affected.

\section{Discussion}

In this study, an experimental model of nephroblastoma was established by transplacental administration of ENU to pregnant rats. The induced tumors were histopathologically compatible with human nephroblastoma. 
Xenotransplant in the nude mouse generated a cultured cell line, ENU-T. A representative clone, ENU-T-1 showed similar characteristics to cultured immature kidney epithelia (Gibson-D'Ambrosio et al. 1987). Therefore, this cell line is of a value to study nephrogenesis as well as nephroblastoma. When transplanted into nude mice, the histological appearance was altered from the original tumor. This phenomenon is common in nephroblastoma cell lines (Hard 1984), which is considered to be the result of defferentiation or selecting-out of organoid-forming clones.

\#11 is considered to bear at least 2 loci for nephroblastoma suppressor genes. Stanbridge and colleagues (Weissmann et al. 1987; Dowdy et al. 1991) have already reported suppressive effect on tumorigenicity of nephroblastoma cells by introduction of \#11 or chromosome fragment including \#11p15 band but not $\# 11$ 13. In this study, introduction of \#11 caused different suppressive effects on CFA and tumorigenicity of rat and human nephroblastoma cell lines. These facts might indicated different pathogenetic mechanisms between human and experimental rat nephroblastomas.

\section{References}

1) Call, K.M., Glaser, T., Ito, C.Y., Buckler, A.J., Pelletier, J., Haber, D.A., Rose, E.A., Kral, A., Yeger, H., Lewis, W.H., Jones, C.H. \& Housman, D.E. (1990) Isolation and characterization of a zinc finger polypeptide gene at human chromosome 11 Wilms' tumor locus. Cell, 61, 509-520.

2) Dowdy, S.F., Fasching, C.L., Araujo, D., Lai, K.-M., Livanos, E., Weissman, B.E. \& Stanbridge, E.J. (1991) Suppression of tumorigenicity in Wilms tumor by the p15.5-p14 region of chromosome 11. Science, 254, 293-295.

3) Gessler, M., Poustka, A., Cavenee, W., Neve, R.L., Orkin, S.H. \& Bruns, G.A.P. (1990) Homozygous deletion in Wilms' tumor of a zinc-finger gene identified by chromosome jumping. Nature, 343, 774-778.

4) Gibsom-D'Ambrosio, R.E., Samuel, M., Chang, C.C., Trosko, J.E. \& D'Ambrosio, S.M. (1987) Characteristics of long-term human epithelial cell cultures derived from normal fetal kidney. In Vitro, 23, 279-287.

5) Hard, G.C. (1984) Tumor biology : In vitro culture and transplantation models of Wilms' tumor. In: Wilms' Tumor, Clinical and Biological Manifestations, edited by C. Prochedley \& E.S. Baun, Elesevier Co., New York, pp. 191-241.

6) Wadey, R.B., Pal, N., Buckle, B., Yeoman, E., Pritchard, J. \& Cewell, J.K. (1990) Loss of heterozygosity in Wilms' tumor involves two distinct regions of chromosome 11. Oncogene, 5, 901-907.

7) Weissman, B.E., Saxon, P.J., Pasquale, S.R., Jones, G.R., Geiser, A.G. \& Stanbridge, E.J. (1987) Introduction of a normal human chromosome 11 into a Wilms' tumor cell line controls its tumorigenic expression. Science, 236, 175-180. 